\title{
A IMPORTÂNCIA DO ESTUDO DA TEORIA DA CARGA COGNITIVA EM UMA EDUCAÇÃO TECNOLÓGICA.
}

\author{
Leila Maria Araújo Santos - Ulbra/SM, PGIE/CINTED/UFRGS \\ leilamas@terra.com.br \\ Liane Margarida Rockenbach Tarouco - CINTED/UFRGS \\ liane@penta.ufrgs.br
}

Resumo: Este artigo descreve a importância do estudo da Teoria da Carga Cognitiva e as contribuições desse para a sociedade contemporânea, que diariamente incorpora novos recursos tecnológicos à educação. Este relato aponta os cuidados que se deve ter ao escolher uma solução tecnológica para apoio a educação, para que esse recurso vá ao encontro do processo cognitivo humano e colabore para um aporte de qualidade ao processo de aprendizagem.

Palavras-chave: Teoria da Carga Cognitiva, objetos de aprendizagem.

Abstract: This article describes the importance of the study of the Theory of the Cognitive Load and the contributions of that for the contemporary society, that daily incorporates new technological resources to the education. This report points the cares that one should have when choosing a technological solution for support the education, for that resource to go to the encounter of the human cognitive process and collaborate for a quality contribution to the learning process.

Keywords: Cognitive Load Theory, learning objects.

\section{A sociedade e a tecnologia}

A história do homem com a natureza é um estado permanente de luta para produzir objetos que transponham as dificuldades impostas pelas forças naturais. $\mathrm{O}$ uso e a fabricação dos mais diferentes instrumentos fez o homem entrar numa dança entre as necessidades naturais e sua própria satisfação. Essa relação entre necessidades e satisfação, do individual ou do coletivo, marca a história do desenvolvimento tecnológico.

No atual estágio de desenvolvimento tecnológico da sociedade contemporânea, as mudanças processam-se em uma velocidade muito grande. Estas mudanças estão ocorrendo no mundo inteiro e em todas as áreas, como afirma Moran: "a sociedade está mudando em todos os países, em todas as instituições, em todos os campos" (2001, p.49).

As transformações sociais, culturais e econômicas, que a evolução tecnológica vem causando ao longo da história, estão muito bem retratadas no livro de Alvin Toffler, "A terceira Onda" (1980). O autor, há mais de vinte anos, narrou as mudanças ocorridas na humanidade, provocadas pelo desenvolvimento tecnológico, explicando-as como ondas de desenvolvimento. 
As tecnologias estão cada vez mais presentes em todos os setores de atividades profissionais, tais como, industrial, comercial, agrícola e dos serviços, da administração, do lazer entre outras. Fazem-nos ganhar consciência de alterações significativas, por exemplo, nos conceitos de espaço (que está encurtado) e de tempo (que está muito acelerado), de centro e de periferia (que se aproximam potencialmente), de vizinhança (que já não se restringe apenas ao prédio, à rua, ao bairro ou à zona em que habitamos ou trabalhamos), de público e de privado (que se perpassam cada vez mais nos nossos quotidianos). As tecnologias, nesta perspectiva, e pela sua universalidade, não são apenas instrumentos de trabalho, de pesquisa ou de comunicação. Elas contribuem para a nossa transformação como humanos e, portanto, para uma alteração profunda na nossa autocompreensão e das relações humanas.

O momento atual reveste-se, deste modo, de grande complexidade apresentando contradições e paradoxos inquietantes. Isso requer, sobre tudo, a necessidade de novos conhecimentos. Uma busca constante por orientações, procedimentos, qualificação, que também, exigem uma maior atenção sobre os processos de interação com a tecnologia. Os quais estão alterando as relações afetivas, de negócios, trabalhos, e em especial a educação.

Este é um enorme desafio para a educação e, em particular, para os professores, podendo-se falar da necessidade de uma mudança do paradigma educacional frente a uma educação tecnológica que já se afirma como realidade.

No uso de tecnologias na educação, a qualidade dos recursos de aprendizagem, das situações que se criam, dos ambientes que se desenvolvem, são essenciais para a ampliação do processo de aprendizagem, do envolvimento e da capacidade de interação dos alunos.

Atualmente, um grande número de recursos tecnológicos e midiáticos encontramse a disposição da educação, mas muitos desses recursos ao invés de agregar qualidade ao processo de ensino e aprendizagem, acabam confundindo, desestimulando ou até mesmo dispersando a atenção dos alunos. Saber escolher, ou construir, um recurso tecnológico que venha ao encontro de uma boa educação passa a ser um desafio para todos os elementos envolvidos na educação de uma geração ávida por todo o tipo de tecnologia.

Diante do volume de informações, dos recursos de interação, de animação, de sons e cores, os quais, muitas vezes, fazem parte das soluções usadas na educação, torna-se importante um conhecimento mais amplo sobre o processo cognitivo humano e de como a Teoria da Carga Cognitiva pode ajudar a potencializar a aprendizagem.

\section{0 processo cognitivo humano e a Teoria da Carga Cognitiva.}

Segundo Nunes \& Giraffa (2003), o processo cognitivo humano refere-se ao estudo do processamento humano de informações, ou seja, o estudo de como os seres humanos percebem, processam, codificam, estocam, recuperam e utilizam as informação. A estrutura cognitiva humana inclui três sistemas de memória: a memória sensorial, a memória de curta duração e a memória de longa duração, as quais trabalham juntas. 
Estudos publicados, por George Miller, em 1956, referiam-se a um "número mágico" $7 \pm 2$. Sobre o qual, o sistema cognitivo humano somente consegue processar um número limitado de informações que variam entre 5 a 9 elementos por vez, ou seja, consegue-se assimilar, de maneira natural e satisfatória, de cinco a nove elementos de informação por vez. Uma vez excedidos esses limites, o raciocínio e a aprendizagem ficam abaixo do desempenho esperado, sobrecarregando a estrutura cognitiva.

Partindo desse pressuposto, pesquisas foram conduzidas por mais de 25 anos, nos quais, com o aporte de pesquisadores internacionais, vem-se expandindo e refinando a regra do $7 \pm 2$, chegando-se, atualmente, a um conjunto compreensível de princípios chamado de Teoria da Carga Cognitiva.

Essa teoria é devida a John Sweller (2003), psicólogo australiano, que dedica-se ao estudo dessa teoria que tem por definição ser um conjunto universal de princípios que resultam em um ambiente de aprendizagem eficiente e que conseqüentemente promovem um aumento na capacidade do processo de cognição humana. Princípios estes que tem como objetivo tornar a interação humana com a tecnologia mais alinhada ao processo cognitivo.

Ela baseia-se em dezenas de estudos e pesquisas experimentais, que comprovam que os usos de seus princípios resultam em ambientes de aprendizagem eficientes e, assim, conduzem a uma aprendizagem competente e melhor. Um ambiente de aprendizagem apropriado, de acordo com princípios da Teoria da Carga Cognitiva, minimiza recursos mentais desnecessários, e em troca disso, coloca-os para trabalhar de modo a maximizarem a aprendizagem.

Essa teoria aplica-se a todos os tipos de conteúdos, todos os tipos de mídias, e à todos os estudantes, visto que, ela tem como fim saber como elabora-se as ferramentas de ensino - texto, imagens e áudio - e aplicá-las à todo o conteúdo de ensino, bem como, as plataformas de aprendizagem a distância, no intuito de potencializar a aprendizagem e desenvolver habilidades flexíveis através da criação e uso de recursos e ambientes de aprendizagem que estejam em sintonia com processo cognitivo humano.

Segundo Sweller (2003), a aprendizagem se dá de maneira melhor quando o processo de informação estiver alinhado com o processo cognitivo humano, ou seja, quando o volume de informações oferecidas ao aluno for compatível com a capacidade de compreensão humana. Assim a Teoria da Carga Cognitiva, apóia-se na impossibilidade natural do ser humano em processar muitas informações na memória a cada momento.

Segundo Mayer (2001), em aplicações multimídias, normalmente, faz-se uso de recursos que utilizam mais de um canal de percepção ao mesmo tempo, como por exemplo, visão e audição, gerando sobrecarga cognitiva que pode levar a desorientação, e até mesmo, ao desestimulo do usuário.

Algumas formas de carga cognitiva são consideradas úteis, enquanto outras desperdiçam recursos mentais. Segundo Mayer (2001b), na elaboração de conteúdos para materiais de ensino, deve-se levar em consideração os três principais tipos de carga cognitiva, que são: Carga cognitiva intrínseca (imposto pela complexidade do conteúdo do material de ensino), Carga cognitiva natural (Relevante) (imposto pelas atividades de ensino que beneficiam o objetivo da aprendizagem), Carga cognitiva externa ao conteúdo 
Irrelevante) (não interfere na construção e automação de esquemas, e, conseqüentemente desperdiça recursos mentais limitados que poderiam ser usados para a auxiliar a carga natural)

Intrínseca, natural e externa ao conteúdo, as formas de cargas cognitivas são adicionais. Tendo em vista que a capacidade mental é limitada, para uma aprendizagem de boa qualidade torna-se necessário um balanceamento dessas cargas, para que o processo de aprendizagem atinja um bom nível de eficiência, como mostra a Figura 1.

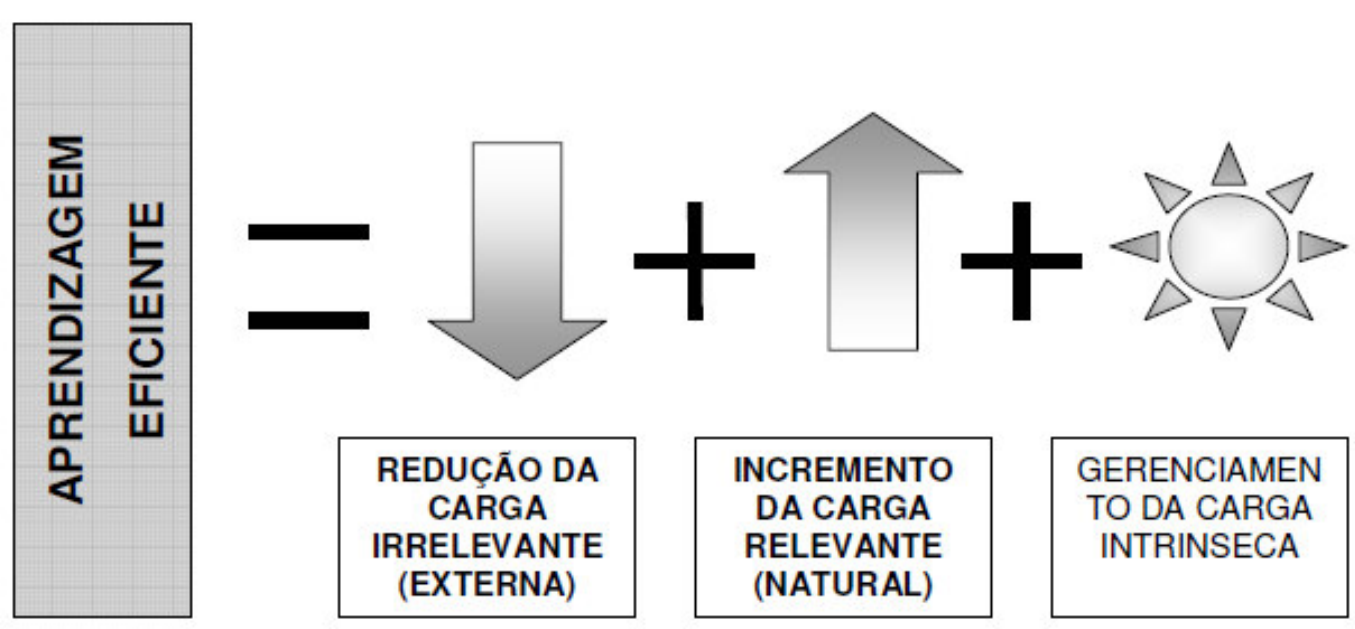

Figura 1. Modelo de balanceamento das cargas cognitivas (Modelo Adaptado de Mayer (2001b)).

Quando não se pode controlar a carga intrínseca associada com os objetivos da aprendizagem, pode-se controlá-la segmentando e arranjando em seqüência o conteúdo de maneira que otimize a quantidade de elementos interativos a qualquer tempo. Por que interatividade significa que diversos elementos do conhecimento devem ser coordenados na memória do aluno para realização de em uma série de atividades e isto pode causar -lhe sobrecarga.

A Teoria da Carga Cognitiva defende que a elaboração de materiais didáticos, principalmente os que utilizam multimídia, deve seguir alguns princípios para, assim, diminuir a sobrecarga cognitiva do aluno e potencializar seu aprendizado. São princípios defendidos por esta teoria:

Princípio de Representação Múltipla: os alunos aprendem melhor quando se combinam palavras e imagens, do que no momento em que se usam somente palavras. 
Princípio de Proximidade Espacial: esse princípio diz respeito à proximidade de palavras e imagens, ou seja, é quando palavras e imagens correspondentes estão próximas em vez de afastadas.

Princípio da Não Divisão ou da Proximidade Temporal: nesse princípio tem se

a apresentação de palavras e imagens simultaneamente em vez de sucessivamente, uma vez que a apresentação de um texto e de uma animação na mesma tela divide a atenção do aluno.

Princípio das Diferenças individuais: sabe-se que estudantes com maior nível de conhecimento, sobre um determinado assunto e com grau maior de orientação espacial possuem maiores condições de organizar e processar seu próprio conhecimento ao interagir com o assunto.

Princípio da Coerência: refere-se à exclusão de palavras, imagens ou sons não relevantes para o assunto. Quanto mais simples e objetiva for à apresentação do conteúdo, mais livre ficará a memória de trabalho para processar um número maior de conhecimentos.

Princípio da Redundância: nesse princípio, ressalta-se que o uso da animação e narração, quando usadas simultaneamente no processo de ensino, potencializa o conhecimento, diferente de quando usadas separadamente.

Estes princípios foram elaborados por Richard Mayer, professor pesquisador da Universidade de Califórnia, Santa Bárbara, dando continuidade à pesquisa sobre a Teoria da Carga Cognitiva de Sweller, e provaram minimizar as sobrecargas cognitivas, potencializando, assim, o processo cognitivo de aprendizagem.

\section{A Importância do Estudo da Teoria da Carga Cognitiva para uso na} educação

Em uma sociedade competitiva, na qual interage-se diariamente com os mais variados recursos tecnológicos, seja em casa, no trabalho ou no lazer, e a valorização do conhecimento está cada vez mais significativa, pode-se fazer uso dos princípios da Teoria da Carga Cognitiva para potencializar o processo de aprendizagem e de interação com a tecnologia.

Não é apenas a educação que se defronta com novas tecnologias: essas estão impactando todo o universo social, e gerando novas dinâmicas, nas quais, o conhecimento torna-se gradativamente central. A transformação envolve praticamente todas as áreas de atividade, economia, política, cultura, a própria organização do tecido social e de relações, além de provocar uma mudança radical na utilização de um recurso não-renovável, o tempo.

A importância do estudo justifica-se no momento em que a sociedade, e os sistemas de gestão do conhecimento que se desenvolvem em torno dela, necessitam aprender a utilizar as novas tecnologias para transformar a educação, na mesma proporção 
em que estas tecnologias estão transformando o mundo que nos cerca. A transformação é de forma e de conteúdo.

Tendo como foco a aprendizagem de seus alunos, os professores que trabalham com tecnologia e contam com os mais variados recursos de interação, tais como, Internet, jogos, objetos de aprendizagem, podem-se valer da Teoria da Carga Cognitiva como um subsídio na escolha dos recursos mais apropriados para sua prática pedagógica. Fazendo assim, uma seleção de recursos que contribuam com o aprendizado de seus alunos. Transformando a interação com a tecnologia além de algo moderno e motivador, em um elemento que realmente potencialize os processos cognitivos dos alunos.

Atualmente, fala-se muito do uso de objetos de aprendizagem na educação.

Segundo Wiley (2002), esses são elementos de um novo tipo de instrução baseada em computador, construído sobre o paradigma da orientação a objetos da ciência da computação. Eles permitem aos desenvolvedores a construção de pequenos componentes instrucionais, modulares, os quais podem ser reutilizados em diferentes contextos de aprendizagem. Os objetos de aprendizagem são visto, por pesquisadores e professores, como um recurso dinâmico e interativo que proporciona um maior interesse de alunos ao processo educacional.

Pensando em popularizar o uso desses objetos, foram criados no Brasil vários repositórios de acesso livre, tais como o Rived (Rede Internacional Virtual de Educação) do Ministério da Educação, o Cesta (Coletânea de Entidades de Suporte ao uso da Tecnologia na Aprendizagem) da UFRGS, onde os interessados podem ter acesso a objetos desenvolvidos sobre variados temas.

No entanto, mesmo o professor tendo acesso a esses objetos, é preciso que o mesmo tenha conhecimentos para avaliar o grau de carga cognitiva presente no recurso escolhido e as reais contribuições que o uso desse fará em sua prática em sala de aula.

Ou, até mesmo, saber se dá maneira como o assunto está sendo apresentado irá potencializar o processo cognitivo do seu aluno.

Recursos multimídia e com interatividade são vistos por professores como forma de motivar o aluno para uma aprendizagem mais eficaz. No entanto, segundo Sweller (2005), em alguns ambientes, estes fatores podem distrair o aluno e causar um impacto negativo no processo de aprendizagem, resultando num aumento da carga cognitiva, ou seja, da quantidade de recursos cognitivos alocados para a realização de uma tarefa específica.

Estudos mostram que a carga cognitiva é um fator sempre presente na interação do homem com o computador, porque cada um dos elementos ou dos objetos da tela deve ser interpretado pelo usuário e conseqüentemente ocupa alguma energia mental do usuário. $\mathrm{O}$ importante é saber escolher um recurso que apresente uma carga reduzida e que possa maximizar o processamento do conhecimento que está sendo ensinado.

Em uma análise realizada em objetos de aprendizagem, disponíveis na Internet, verificou-se em muitos deles um cuidado com o layout e com o conteúdo apresentado. 
Em sua maioria são bastante lúdicos e intuitivos, mas seguindo os princípios da Teoria da Carga Cognitiva, apresentam sobre-posição de elementos, o que leva a sobrecarga cognitiva, e como resultado um baixo desempenho do processo cognitivo.

Alguns apresentam carga cognitiva externa, que é a carga cognitiva relacionada a elaboração do objeto, causada pela superposição de texto e a narração do mesmo, o que segundo a teoria causa uma sobrecarga cognitiva em função de ocupar dois canais de percepção (visão e audição) ao mesmo tempo e com informações redundantes, o que pode interferir negativamente no processo de aprendizagem.

Também se verificou a presença de uma sobrecarga, causada pela narração contínua de atividades, ou seja, a narração não é interrompida quando é selecionada outra atividade, ficando a narração da atividade anterior como pano de fundo de outra atividade, o que pode causar confusão no aluno.

Percebe-se, também, que em alguns objetos de aprendizagem a carga cognitiva é intrínseca, ou seja, relacionadas com o conteúdo apresentado, pois, em alguns casos, existe um excesso de informações redundantes, e, também, atividades que não oferecem o feedback aos alunos, o que pode induzi-los a achar que realizaram acertadamente as atividades.

Segundo Tarouco (2006), a carga cognitiva externa pode ser minimizada pelo projetista do objeto, observando os princípios da teoria, já que em uma interface complexa ou não-convencional que usa diferentes fontes, objetos, ferramentas da navegação, e padrões de layout terá geralmente uma carga cognitiva elevada porque cada componente necessitará ser percebido e interpretado pelo aprendiz. Uma interface que use convenções padrão no texto, gráficos, navegação e layout será mais facilmente interpretada e consequientemente terá uma carga cognitiva muito mais baixa. Já a carga cognitiva interna, derivada do conteúdo em si, depois do objeto de aprendizagem pronto não pode ser reduzida, a não ser pela segmentação do material contido em um objeto de aprendizagem.

Também é necessário avaliar de forma apropriada à combinação dos diversos tipos de mídia disponibilizados pela tecnologia. Estudos recentes mostram que o sistema de cognição humana dispõe de entradas independentes para a informação visual e para a verbal. E ao se fazer uso de dois canais de percepção independentes no processo de ensino e aprendizagem, a carga cognitiva pode ser reduzida.

Segundo Mayer (2002), a redução da carga em decorrência do uso de dois canais de percepção ocorre apenas quando a informação presente nas diferentes modalidades não é redundante, pois ao contrário a carga cognitiva aumenta, ou seja, o uso de dois canais de percepção com objetivos complementares, como por exemplo, uma imagem e a narração de um texto, e não com objetivo de repetição, como por exemplo, um texto escrito e a narração do mesmo.

O objetivo de um bom layout para uso educacional é reduzir a quantidade de energia direcionada à interação com o sistema, liberando assim a capacidade cognitiva para o processamento do que está sendo ensinado. 


\section{Considerações Finais}

Os materiais educacionais que fazem uso de recursos digitais freqüentemente sobrecarregam a memória de trabalho dos aprendizes e dificultam a aquisição de esquemas que requer reflexão. Uma análise baseada nos princípios da Teoria da Carga Cognitiva só reforça a idéia de que os recursos tecnológicos por mais atraente que possam parecer, nem sempre estão de acordo com o processo cognitivo humano e por isto nem sempre qualificam o processo de aprendizagem. Por isso, esse artigo destaca a necessidade de uma maior divulgação da Teoria da Carga Cognitiva à educadores para que possam fazer uso da tecnologia na educação mais adequada ao desenvolvimento educacional de seus alunos.

O estudo da Teoria da Carga Cognitiva serve como base par a o desenvolvimento de tese de doutorado em Informática na Educação a qual que tem como objetivo aliar os princípios dessa teoria ao planejamento, elaboração e análise de recursos tecnológicos de apoio à educação.

\section{Bibliografia}

CARVALHO, Ana A.A. A Teoria da Flexibilidade Cognitiva na Formação à Distancia: um estudo da www. (2002) Disponível em: $<$ http://event.ua.pt/1siie99/espanhol/pdfs/comunicacao03.pdf.> Acessado em 08 maio de 2006. ress. 2001

MAYER, Richard. Multimedia Learning. Cambridge: Cambridge University

Cognitive Constraints on Multimedia Learning: When Presenting More Material Results in Less Understanding. Journal of Educational Psychology. Vol. 93, $\mathrm{N}^{\circ} 1,187-198.2001 \mathrm{~b}$.

; MORENO, R. Animation as an Aid to Multimedia Learning. Educational Psychology Review, V.14, N.1, March 2002

MORAN. José M. Novos desafios na educação: a internet na educação presencial e virtual. In: PORTO, Tania M. E. (Org.). Saberes e linguagens de educação e comunicação. Pelotas: Ed. Universitária, 2001.

NUNES, Marcelo; GIRAFFA, Lúcia. A educação na ecologia digital. PPGCC/ FACIN, PUCRS, 2003.

SWELLER, John. Cognitive Load Theory: A Special Issue of educational Psychologist”. LEA, Inc, 2003.

SWELlER, J.; MERRIENBOER, J. Cognitive Load Theory and Complex earning: Recent Developments and Future Directions. Educational Psychology Review, V.17, N.2, June 2005. 
TAROUCO, L; Cunha, S. Aplicação de teorias cognitivas ao projeto de objetos de aprendizagem. V. $4 \mathrm{~N}^{\circ}$ 2, Dezembro, 2006. CINTED/UFRGS. Porto Alegre-RS. Disponível em < http://www.cinted.ufrgs.br/renote/dez2006/artigosrenote/25025.pdf > Acesso junho de 2007.

WILEY, D. The instructional use of learning objects. 2002. Disponível em: $<$ http://www.reusability.org/read/>. Acesso em Março de 2007. 